\title{
Assessment of image-derived risk factors for natural course of unruptured cerebral aneurysms
}

\author{
Manasi Ramachandran, PhD, ${ }^{1}$ Rohini Retarekar, PhD, ${ }^{1}$ Madhavan L. Raghavan, PhD, ${ }^{1}$ \\ Benjamin Berkowitz, MS, ${ }^{1}$ Benjamin Dickerhoff, MS, ${ }^{1}$ Tatiana Correa, BSE, ${ }^{1}$ Steve Lin, BS, ${ }^{1}$ \\ Kevin Johnson, BSE, ${ }^{2}$ David Hasan, MD, ${ }^{2}$ Christopher Ogilvy, MD, ${ }^{3}$ Robert Rosenwasser, MD, ${ }^{4}$ \\ James Torner, PhD, ${ }^{5}$ Einar Bogason, MD, ${ }^{6}$ Christopher J. Stapleton, MD, ${ }^{7}$ and \\ Robert E. Harbaugh, MD ${ }^{6,8}$
}

'Department of Biomedical Engineering, University of lowa; ${ }^{2}$ Department of Neurosurgery, University of lowa Hospitals and Clinics; and ${ }^{5}$ Department of Epidemiology, University of lowa, lowa City, lowa; ${ }^{2}$ Department of Surgery, Division of Neurosurgery, Beth Israel Deaconess Medical Center; and 'Department of Neurosurgery, Massachusetts General Hospital, Boston, Massachusetts; ${ }^{4}$ Department of Neurosurgery, Jefferson University Hospital, Philadelphia; ${ }^{6}$ Department of Neurosurgery, Penn State University, Hershey; and ${ }^{8}$ Department of Engineering Science and Mechanics, Pennsylvania State University, State College, Pennsylvania

OBJECTIVE The goal of this prospective longitudinal study was to test whether image-derived metrics can differentiate unruptured aneurysms that will become unstable (grow and/or rupture) from those that will remain stable.

METHODS One hundred seventy-eight patients harboring 198 unruptured cerebral aneurysms for whom clinical observation and follow-up with imaging surveillance was recommended at 4 clinical centers were prospectively recruited into this study. Imaging data (predominantly CT angiography) at initial presentation was recorded. Computational geometry was used to estimate numerous metrics of aneurysm morphology that described the size and shape of the aneurysm. The nonlinear, finite element method was used to estimate uniform pressure-induced peak wall tension. Computational fluid dynamics was used to estimate blood flow metrics. The median follow-up period was 645 days. Longitudinal outcome data on these aneurysm patients-whether their aneurysms grew or ruptured (the unstable group) or remained unchanged (the stable group) - was documented based on follow-up at 4 years after the beginning of recruitment.

RESULTS Twenty aneurysms (10.1\%) grew, but none ruptured. One hundred forty-nine aneurysms (75.3\%) remained stable and 29 (14.6\%) were lost to follow-up. None of the metrics-including aneurysm size, nonsphericity index, peak wall tension, and low shear stress area-differentiated the stable from unstable groups with statistical significance.

CONCLUSIONS The findings in this highly selected group do not support the hypothesis that image-derived metrics can predict aneurysm growth in patients who have been selected for observation and imaging surveillance. If aneurysm shape is a significant determinant of invasive versus expectant management, selection bias is a key limitation of this study.

http://thejns.org/doi/abs/10.3171/2015.2.JNS142265

KEY WORDS aneurysm; quantified aneurysm shape; subarachnoid hemorrhage; aneurysm growth; vascular disorders

I DENTIFYING risk factors that determine the natural history of unruptured intracranial aneurysms could aid in their clinical management. Morphological characteristics are candidates for such risk factors, because morphology varies in the patient population, the variation can be measured, and morphological variations affect the aneurysm's biomechanical environment (e.g., pressure- induced wall tension and flow-induced shear) in ways that may cause aneurysm enlargement and rupture. In addition, the shape of the aneurysm may reflect the underlying biology of the aneurysm wall. Numerous studies have assessed the differences between unruptured and ruptured aneurysms in regard to morphology, $4,13,16,20,23,33,35,37$ blood flow, ${ }^{4,6,7,12,22,31,32,37}$ and wall tension. ${ }^{18}$ Such studies have

ABBREVIATIONS CE = contrast enhanced; CTA = CT angiography; Dmax = maximum diameter; $L S A=$ low shear area; $M R A=$ MR angiography; NSI = nonsphericity index; PWT = peak wall tension; TOF = time of flight.

ACCOMPANYING EDITORIAL See pp 285-287. DOI: 10.3171/2015.4.JNS15465.

SUBMITTED October 3, 2014. ACCEPTED February 27, 2015.

INCLUDE WHEN CITING Published online September 18, 2015; DOI: 10.3171/2015.2.JNS142265. 
found that key aspects of aneurysm shape and blood flow reliably differentiate between ruptured and unruptured aneurysms. However, the ability to differentiate between ruptured and unruptured aneurysms may not translate into an ability to distinguish whether a particular unruptured aneurysm is destined to enlarge or rupture.

In a 2012 report on the natural course of unruptured aneurysms, the UCAS (Unruptured Cerebral Aneurysms) Japan investigators reported that aneurysms with a daughter sac were more likely to rupture. ${ }^{19}$ In a retrospective case-control study, Takao et al..$^{32}$ noted that 6 internal carotid artery aneurysms that ruptured during observation had a lower minimum wall shear stress than 44 locationmatched control cases. In a study of 7 unruptured intracranial aneurysms that were noted to have grown under observation, Boussel et al. ${ }^{5}$ reported that regions of low wall shear stress correlated with regions of growth. These reports suggest the possibility that image-derived metrics such as morphology, blood flow, and wall tension could serve as risk factors for growth and rupture of unruptured aneurysms under observation.

The goal of this prospective investigation was to use 3D image processing and computational methods to determine whether metrics of morphology, blood flow, and wall tension of unruptured cerebral aneurysms could distinguish between those that would go on to grow and/or rupture (unstable) and those that would remain unchanged (stable), in a group of patients with unruptured aneurysms for whom observation and surveillance imaging were recommended.

\section{Methods \\ Study Population}

The study population consisted of 178 consecutive patients harboring 198 unruptured aneurysms for whom observation and surveillance imaging were the recommended management and adequate imaging was available. Patients had to have adequate diagnostic studies and had to consent to this prospective study at 4 participating clinical centers: Penn State Hershey Medical Center (clinical coordinating center), Massachusetts General Hospital, Jefferson University Hospital, and University of Iowa Hospitals and Clinics. Data analysis investigators at the University of Iowa (image analysis center) were blinded to outcome information until all the computational analyses were completed. Institutional review board approvals were obtained at all participating clinical centers and the data analysis center.

Aneurysms in the study population encompassed all the common locations reported in the literature. Table 1 has tabulated locations and Fig. 1 is a visual representation of the precise locations and orientations within vessels. Most aneurysms were located on the internal carotid arteries and the middle cerebral arteries, which is consistent with data reported by the International Study of Unruptured Intracranial Aneurysms. ${ }^{36}$ All were saccular aneurysms because only patients with saccular aneurysms were considered for the study. Study participants were followed longitudinally according to routine clinical practice at the participating centers, typically at 6 -month to
TABLE 1. Demographic data for study population in the stable and unstable groups

\begin{tabular}{|c|c|c|c|}
\hline Characteristic & Stable & Unstable & All \\
\hline No. & 149 & 20 & 169 \\
\hline Median age, yrs & 63 & 61 & 63 \\
\hline $\operatorname{Sex}(\% F)$ & $81 \%$ & $80 \%$ & $81 \%$ \\
\hline Race (\% white) & $89 \%$ & $83 \%$ & $89 \%$ \\
\hline Smoking history & $58 \%$ & $65 \%$ & $59 \%$ \\
\hline Hypertension & $50 \%$ & $55 \%$ & $51 \%$ \\
\hline \multicolumn{4}{|l|}{ Scan modality } \\
\hline CTA & 117 & 15 & 132 \\
\hline CE MRA & 7 & 0 & 7 \\
\hline TOF MRA & 25 & 5 & 30 \\
\hline Median follow-up, days & 607 & 743 & 645 \\
\hline Multiple constellations & 46 & 2 & 48 \\
\hline \multicolumn{4}{|l|}{ Location } \\
\hline ICA & 49 & 8 & 57 \\
\hline MCA & 45 & 2 & 47 \\
\hline $\mathrm{ACoA}$ & 18 & 3 & 21 \\
\hline ACA & 13 & 2 & 15 \\
\hline BA & 7 & 1 & 8 \\
\hline OphA & 5 & 1 & 6 \\
\hline PCoA & 2 & 2 & 4 \\
\hline PCA & 3 & 0 & 3 \\
\hline SCA & 2 & 1 & 3 \\
\hline VA & 2 & 0 & 2 \\
\hline PICA & 2 & 0 & 2 \\
\hline Para-OphA & 1 & 0 & 1 \\
\hline
\end{tabular}

$\mathrm{ACA}=$ anterior cerebral artery; $\mathrm{ACo}=$ anterior communicating artery; $\mathrm{BA}$ = basilar artery; ICA = internal carotid artery; $M C A=$ middle cerebral artery; OphA = ophthalmic artery; $\mathrm{PCA}=$ posterior cerebral artery; $\mathrm{PCoA}=$ posterior communicating artery; PICA = posterior inferior cerebellar artery; SCA = superior cerebellar artery; $\mathrm{VA}=$ vertebral artery.

1-year intervals. In September 2011, a data analysis investigator visited each clinical site, collected aneurysm status information (i.e., "grown," "ruptured," "shrunk," and "unchanged"), and verified it against documented clinical notes with the clinical coordinator at each site. The study definition of aneurysm growth was based on review of images by expert clinicians, not by quantified assessment of aneurysm surface area, volume, or other metrics. Although this inevitably introduces subjectivity in this outcome measure, we believe that this represents the realworld methodology used to determine if an unruptured aneurysm has shown evidence of growth. As a post hoc analysis of study data we used a quantitative threshold to define growth, and we repeated all our statistical analyses using this definition. This post hoc analysis is described in the Discussion section.

\section{Image Data Acquisition and Aneurysm Reconstruction}

Volumetric diagnostic scans of the aneurysms at initial presentation were de-identified and transferred to the data analysis site as they became available. Computed tomography angiography (CTA) was the predominant scan mo- 


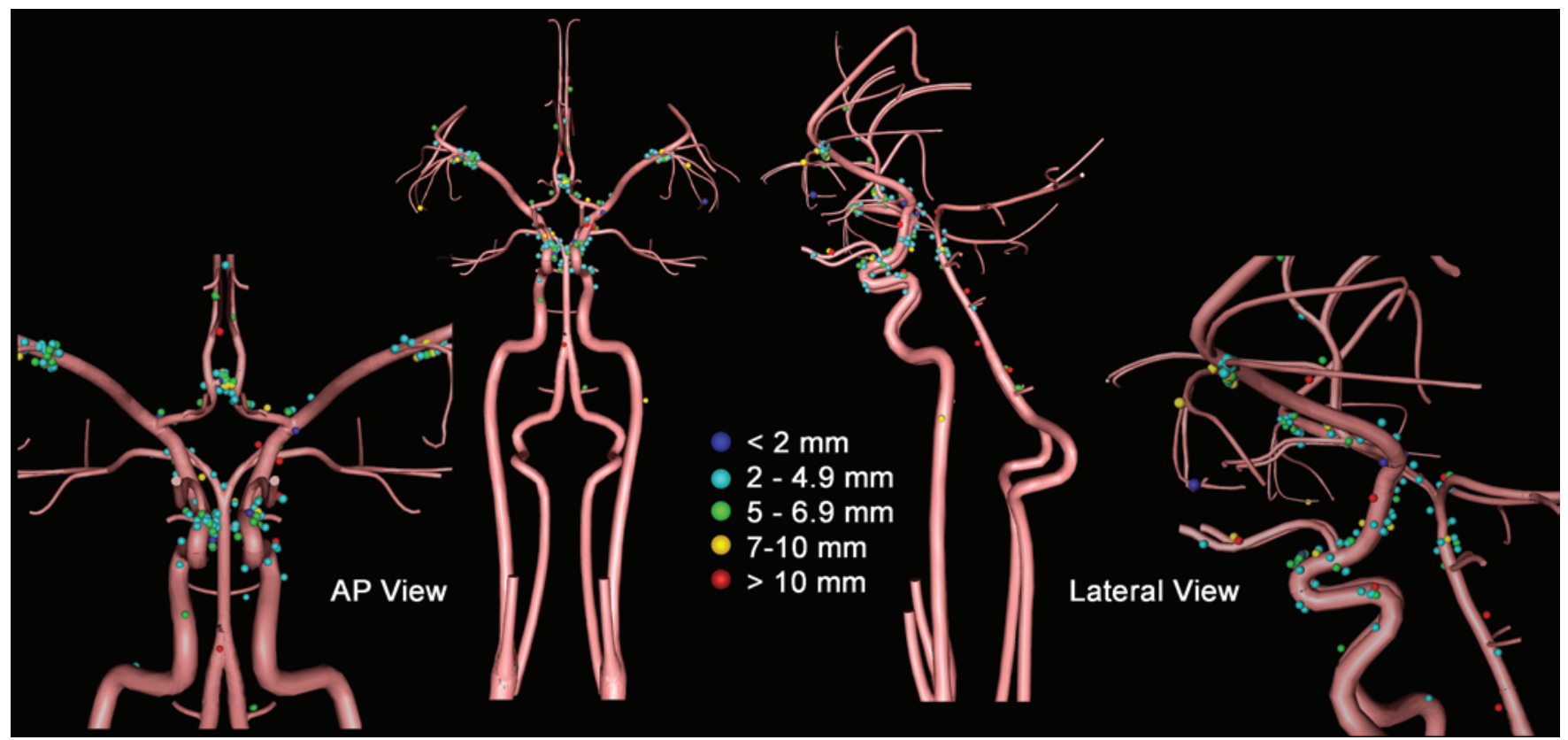

FIG. 1. Graphical illustration of the locations, orientation, and sizes (see color legend) of all aneurysms in patients recruited in this study, on an idealized 3D model of the head and neck arteries. Table 1 provides location-specific counts in this study. AP = anteroposterior.

dality (78\%), but scans for some aneurysms were by timeof-flight magnetic resonance angiography (TOF MRA; $18 \%$ ) and contrast-enhanced (CE) MRA (4\%). Image resolution was $0.41 \mathrm{~mm}$ on average (range $0.20-0.86 \mathrm{~mm}$ ), and slice spacing ( $\mathrm{Z}$ resolution) was $0.64 \mathrm{~mm}(0.30-1.00 \mathrm{~mm})$. A protocol was developed for the $3 \mathrm{D}$ reconstruction of the aneurysm from DICOM image data by using level set segmentation techniques, ${ }^{2}$ as implemented in the Vascular Modeling ToolKit (VMTK; open source software). The parent vasculature and the aneurysm were reconstructed using level set initialization methods; colliding fronts and fast marching, respectively. ${ }^{1,2,21}$ The reconstruction protocol was verified using a flow phantom with a $10-\mathrm{mm}$ anterior communicating artery aneurysm, as reported by us earlier. ${ }^{26}$ Four data analysis investigators were trained, first using flow phantom image datasets and then using image volume datasets from 5 patients' aneurysms. Detailed descriptions of the image processing protocol may be found in our earlier publications. . $^{13,24,25}$

\section{Determination of Aneurysm Metrics}

Metrics that capture the geometry, blood flow, and pressure-induced wall tension of aneurysms were computed from the reconstructed 3D models. For computing most geometrical metrics, the aneurysm sac was isolated using a single cutting plane-the neck plane-as described in our earlier reports. ${ }^{13,23}$ The neck plane was chosen by a single user for all the aneurysms in the study population to minimize investigator sensitivity. Size and shape metrics that were developed by our group and have been described in our earlier reports ${ }^{17,23}$ were computed (Table 2). Also included was size ratio, as reported by Xiang et al. ${ }^{37}$ (sac size/vessel size) and shown to distinguish rupture status. Maximum diameter (Dmax) ${ }^{23}$ was used for sac size. Parent vessel size was defined as the diameter of the largest inscribed sphere inside the parent vessel closest to the sac, as implemented in the sac isolation algorithm in Ford et al. ${ }^{11}$

Using the isolated sac, we also performed nonlinear finite element analysis to compute the pressure-induced wall tension (stress resultant) distribution under a consistent pressure of $120 \mathrm{~mm} \mathrm{Hg}$ for all aneurysms with the aneurysm neck constrained from displacement, as reported in our earlier work. ${ }^{25}$ Wall tension in the aneurysm sac is affected by sac size, sac shape, and blood pressure. We had earlier shown that for all practical purposes, choice of material behavior has no observable effect in stratifying a study population like this one based on wall tension. In this study, a 2-parameter polynomial finite elastic constitutive model with spatially uniform parameter values and wall thickness of $86 \mu \mathrm{m}$ was used, as detailed in that report. One metric of wall tension was used-the peak wall tension (PWT)-defined as the 95th percentile value of maximum principal wall stress multiplied by the wall thickness of $86 \mu \mathrm{m}$ (to obtain the stress resultant). In an earlier report ${ }^{9}$ on a similar longitudinal study of rupture risk in infrarenal aortic aneurysms, we showed that PWT was a better predictor than aneurysm diameter. The use of uniform $120 \mathrm{~mm} \mathrm{Hg}$ pressure consistently for all aneurysms essentially means that the PWT metric is merely a latent metric of aneurysm sac surface morphology, but it serves the purpose of combining the effect of both sac size and sac shape in a manner that is consistent with the physics of hemodynamics.

Finally, using the aneurysm sac and its contiguous vasculature, blood flow metrics were also computed. Computational fluid dynamics simulations were performed on 3D models using the commercial software Fluent (Ansys, Inc.). A finer computational mesh was used in regions of sharper curvature such as the aneurysm neck. ${ }^{28}$ Triangular 


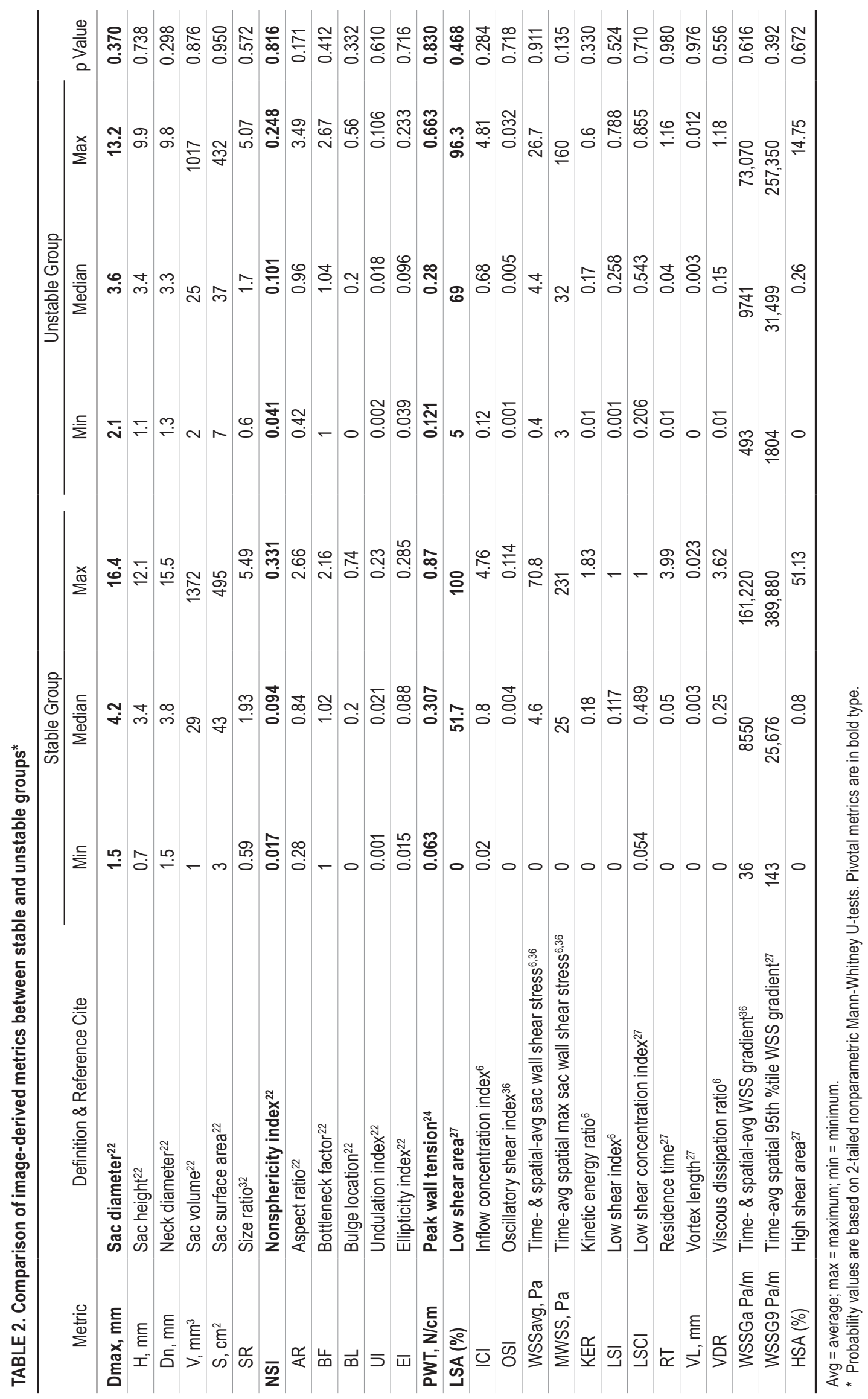


and tetrahedral elements were used to mesh the surface and volume, respectively. To capture the boundary layer with better accuracy, prism elements were used to mesh the near wall region. A velocity input boundary condition was implemented by using descriptive statistics of the internal carotid waveform presented by Ford et al. ${ }^{10}$ To minimize entrance effects, cylindrical extensions were added to the inlets. At the outlets, a constant pressure boundary condition was implemented. A slip boundary condition was not applied and blood was assumed to behave as a Newtonian, incompressible fluid. Two cardiac cycles were simulated for each patient and results from the second cycle were used for quantification of flow behavior. For the simulation, a time step size of 0.001 seconds was used and 300 iterations were performed for each time step. To calculate hemodynamic indices, aneurysms were isolated from the contiguous vasculature by using cutting planes. Blood flow-based indices reported by other research groups, $7,12,37$ with minor modifications to their definitions as reported by us in a recent publication, ${ }^{28}$ were used to quantify the nature of flow patterns inside the aneurysm. A detailed technical description of our computational modeling may be found in earlier reports. ${ }^{23,24,28}$ Table 2 summarizes the various geometrical, wall tension, and blood flow metrics computed in this study.

\section{Statistical Treatment}

Aneurysms used in the study were placed in 1 of 2 groups depending on their follow-up status-unstable group ("grown" or "ruptured") and stable group ("unchanged" or "shrunk"). Prior to performing statistical analysis comparing the 2 groups, we identified a pivotal metric for each of the metric types that captures its essence based on factors such as robustness of definition, clarity of physical meaning, exhaustiveness, and likelihood for being a risk factor based on previous reported studies on rupture status. For size we chose Dmax instead of volume, because of its familiarity in the clinical realm. For shape we chose nonsphericity index (NSI), mainly because of its exhaustive nature in capturing sac shape. For wall tension we chose PWT, because aneurysm rupture may be thought to occur at a single spatial location when elevated wall tension at a site exceeds the wall's ability to withstand it. For blood flow we chose percent low shear area (LSA), because its definition is robust (its range is bounded from 0 to $100 \%$ ), it has a clear physical meaning, it is probably associated with sac wall inflammation, and reports in the literature suggest that it may be related to rupture status and growth risk. ${ }^{5,12,14,37}$ The a priori hypotheses were that these 4 pivotal metrics would be different between the groups, and these were tested first, after which all other metrics were tested for predictability post hoc.

\section{Results}

The 198 aneurysms were followed for a median period of 645 days. Over the follow-up period, none ruptured, $20(10.1 \%)$ had grown, $149(75.3 \%)$ were found to be stable, and $29(14.6 \%)$ were lost to follow-up. Fifty-five $(27.8 \%)$ aneurysms were treated during the observation period. Reasons for treatment included identification of growth, patient requested treatment during observation, or a change in management strategy by the treating physicians. Sac size, sac shape, wall tension, and blood flow metrics were calculated for all aneurysms. The ShapiroWilk ${ }^{30}$ normality test (Statistical software package; SPSS) showed that all the metrics had nonnormal distributions. Thus, the nonparametric Mann-Whitney U-test was used. Statistical significance was defined as 2 -tailed $\mathrm{p}<0.05$. Comparisons between the stable and unstable groups are tabulated in Table 1 (demographics), Table 2 (metrics), and Fig. 2. The pivotal metrics did not differentiate the 2 groups: Dmax $(\mathrm{p}=0.370)$; NSI $(\mathrm{p}=0.816) ;$ PWT $(\mathrm{p}=$ $0.830)$; and LSA $(p=0.468)$. None of the other metrics individually differentiated the 2 groups on post hoc analysis, including demographic and medical history variables such as age, sex, and hypertension.

\section{Discussion}

Aneurysm morphology is an obvious candidate as a
Dmax, mm

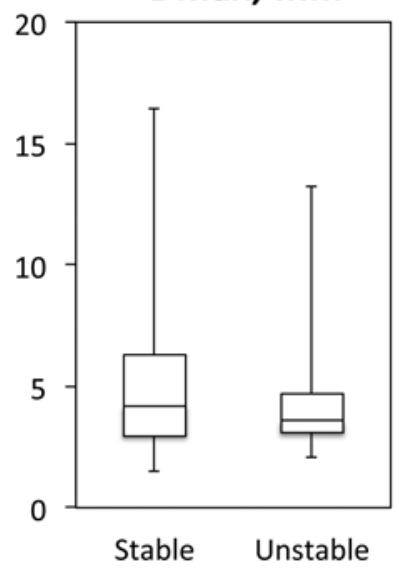

NSI

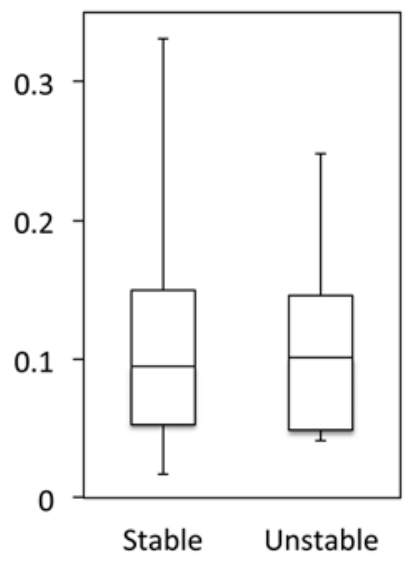

PWT, N/cm

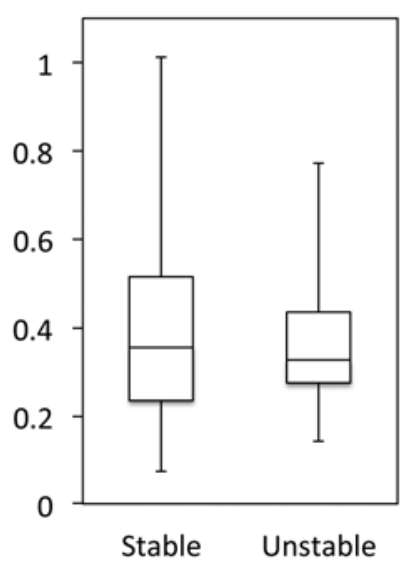

LSA, \%

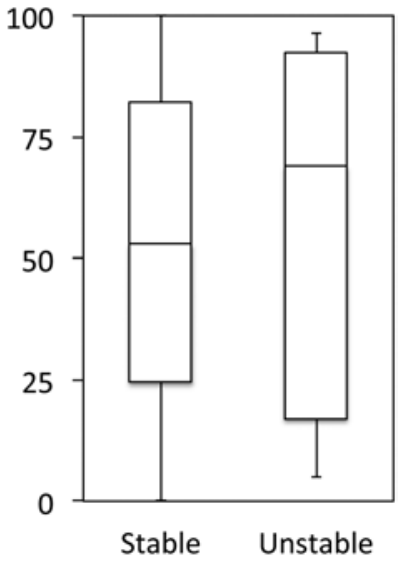

FIG. 2. Quartiles comparing pivotal metrics (Dmax, NSI, PWT, and LSA) at the initial presentation in unruptured aneurysms that remained stable during observation and those that became unstable. Box denotes the 25th, median, and 75th percentile values, and whiskers represent the maximum and minimum. 
risk factor for instability. Studies that compared ruptured to unruptured aneurysms have identified many risk factors that are either direct (e.g., NSI and size ratio) ${ }^{8,23,37}$ or latent (e.g., inflow concentration index, ${ }^{7} \mathrm{LSA}^{37}$ ) measures of morphology. The UCAS Japan investigators found that aneurysms with daughter sacs were at a higher risk of rupture. ${ }^{19}$ This study was designed to test the hypothesis that morphology would help differentiate aneurysms at a high risk of instability in a prospectively recruited population. Many aspects of this study design were unique for their rigor, such as prospective recruitment; longitudinal followup rather than case-control; a focus on aneurysm growth risk rather than rupture status; blinding of data analysis investigators from outcome data; restraints against sneak peeks into study findings; state-of-the-art image processing and computational approaches; objective computations of metrics; and the inclusion of metrics that encompass aneurysm geometry, tissue mechanics, and flow mechanics.

Our study's lack of support for the hypothesis that morphological metrics at initial presentation could differentiate between aneurysms that will become unstable and those that will remain stable stands in contrast to inferences drawn from a large body of earlier studies in the literature that have compared aneurysms that presented with a rupture with those that presented unruptured. $3,7,8,14,23,33,37$ For instance, blood flow metrics have been suggested as good predictors for aneurysm rupture. To further explore whether any differences in flow metrics could predict aneurysm behavior, we conducted a controlled test by choos- ing a location and size-matched control from the stable group for each of the aneurysms in the unstable group (i.e., same location, including bifurcation versus sidewall, and with a size as close as possible). No consistent differences were found between the unstable aneurysms and their stable counterparts in the metrics that capture the essence of the flow characteristics (Fig. 3). These paired comparisons did not differ from the overall findings of the study.

This study's results need to be seen within the context of limitations inherent in such longitudinal studies and limitations that may be addressed with further efforts.

\section{Selection Bias}

We believe that it is highly likely that a selection bias exists in this study population because physicians with expertise in management of patients with unruptured intracranial aneurysms are more likely to recommend invasive treatment for aneurysms with larger size and irregular shape. In this study of patients with unruptured aneurysms for whom observation rather than invasive treatment was recommended, the NSI, a metric that captures the deviation from spherical shape of the aneurysm sac, was 0.11 \pm 0.06 (mean $\pm \mathrm{SD}$ ). A perfect hemisphere sac will have an NSI of 0 , and the NSI increases up to a maximum of 1 as the aneurysm becomes more irregular. In all earlier reports of patients with unruptured aneurysms for whom invasive treatment was recommended,,$^{13,23,35,37}$ the aneurysms had mean NSI values greater than those in our study. In
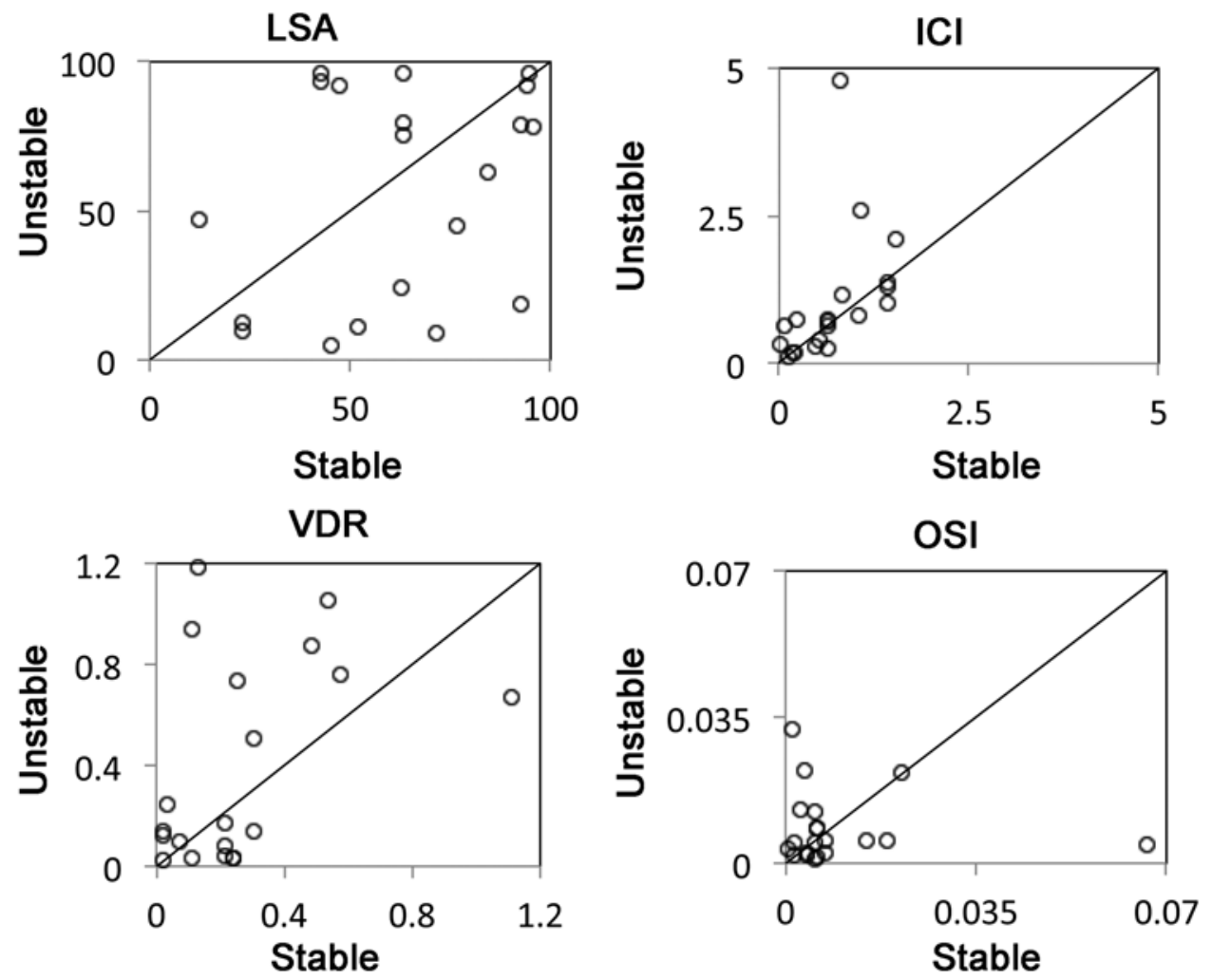

FIG. 3. Scatterplots showing a comparison of flow metrics between representative unstable aneurysms (y axis) and their locationand size-matched paired counterparts in the stable group ( $\mathrm{x}$ axis). Unstable aneurysms do not appear to be consistently higher or lower than stable aneurysms for all these metrics. ICI = inflow concentration index; OSI = oscillatory shear index; VDR = viscous dissipation ratio. 
addition, the aneurysms in this study were, on average, smaller than those in earlier reports..$^{13,23,35,37}$

It is likely that the cerebrovascular experts, who carefully review the available data before making a management decision, include larger aneurysm size and more irregular aneurysm shape as important variables. During the enrollment period for this study, 295 patients with unruptured intracranial aneurysms were screened by the investigators at the Penn State Milton S. Hershey Medical Center and the University of Iowa. These data are not available for the other centers involved in the study. $\mathrm{Ob}-$ servation was recommended for 212 (71.9\%) of the 295 patients who were screened, and 67 patients $(22.7 \%)$ were included in the study. The study group therefore may not be representative of the overall patient population in regard to aneurysm geometry. A separate study is underway to determine if the patients for whom invasive treatment was recommended harbored aneurysms whose geometrical indices were significantly different from the geometrical indices of aneurysms in patients for whom observation and surveillance imaging was recommended.

\section{Definition of Growth}

The fact that none of the aneurysms ruptured in this study during observation meant that the study only assessed growth risk, not rupture risk. How much growth is enough to be termed "unstable" cannot be defined with precision. For example, image artifacts and noise may affect the precision with which follow-up measurements can be compared with earlier imaging studies. As a secondary arm of this study, we performed a post hoc analysis using a more quantitative process to define growth. In a single-center, single-modality (CE MRA) study, Saloner et al. ${ }^{29}$ reported on using volume change as a criterion for growth, verified against 3 clinical investigators' measurements, and proposed that a 5\% change in volume may be a good threshold for defining growth. Because ours is a multicenter, multimodality study, we chose double that $-10 \%$ volume growth-as the criterion. In 18 of the 20 unstable aneurysms, 3D volumetric image data were available after the aneurysms had been determined to have grown based on clinician assessment of the imaging studies. Of these 18 aneurysms, 14 showed greater than $10 \%$ objectively determined volumetric growth. These were categorized as the so-called quantitatively unstable group. For verification, we repeated all statistical tests comparing the quantitatively unstable group $(\mathrm{N}=14)$ with the stable group $(\mathrm{N}=149)$. Once again, none of the metrics differentiated between the groups at a statistically significant level.

\section{Other Limitations}

Unlike many studies on rupture status, in which image data tends to be 3D rotational angiography, the image data in this study were from multiple centers and multiple modalities: predominantly CTA, but also TOF MRA and CE MRA (see Table 1). These modalities have somewhat lower spatial resolution than 3D rotational angiography. This fact and the fact that unruptured aneurysm patients for whom observation was recommended harbored smaller aneurysms creates a poorer signal-to-noise ratio for our study. Although this study assessed a wide range of metrics-including geometry, tissue mechanics, and fluid mechanics-it still did not include all metrics reported in the literature. Some morphometric examples not assessed are the elegant aggregate metrics to characterize sac shape reported by Lauric et al., ${ }^{15,16}$ Valencia et al., ${ }^{35}$ and Piccinelli et al. ${ }^{20}$ For flow characterization, alternative metrics have been reported by Xiang et al., ${ }^{37}$ Valen-Sendstad, ${ }^{34}$ and Lauric et al. ${ }^{14}$ Finally, our study population, although the largest to date and powered to test the pivotal metrics, may not be large enough for the complete list of metrics assessed in post hoc analyses.

\section{Conclusions}

The findings of this study do not support the hypothesis that image-derived metrics are predictive of aneurysm growth in the subset of unruptured cerebral aneurysms evaluated in the current study. This suggests that after a decision is made that observation and imaging surveillance is the best management option, the computational analysis of aneurysm geometry used in this study may not help to predict which aneurysms are likely to enlarge and rupture during observation.

\section{Acknowledgments}

This study was funded by National Heart Lung and Blood Institute grant no. R01HL083475, "Assessment of intracranial aneurysm shape as an indicator of rupture risk." The work described in this manuscript is based on Ph.D. dissertations by the co-lead authors, Ramachandran ${ }^{24}$ and Retarekar. ${ }^{27}$ We are grateful to Deborah Hoffman, study clinical coordinator at Penn State Milton S. Hershey Medical Center, for collection and organization of clinical data. We are grateful to the Vascular Modeling ToolKit (VMTK) developer and user community for assistance with image processing.

\section{References}

1. Antiga L, Ene-Iordache B, Caverni L, Cornalba GP, Remuzzi A: Geometric reconstruction for computational mesh generation of arterial bifurcations from CT angiography. Comput Med Imaging Graph 26:227-235, 2002

2. Antiga L, Ene-Iordache B, Remuzzi A: Computational geometry for patient-specific reconstruction and meshing of blood vessels from MR and CT angiography. IEEE Trans Med Imaging 22:674-684, 2003

3. Bacigaluppi S, Piccinelli M, Antiga L, Veneziani A, Passerini $\mathrm{T}$, Rampini $\mathrm{P}$, et al: Factors affecting formation and rupture of intracranial saccular aneurysms. Neurosurg Rev 37:1-14, 2014

4. Baharoglu MI, Schirmer CM, Hoit DA, Gao BL, Malek AM: Aneurysm inflow-angle as a discriminant for rupture in sidewall cerebral aneurysms: morphometric and computational fluid dynamic analysis. Stroke 41:1423-1430, 2010

5. Boussel L, Rayz V, McCulloch C, Martin A, Acevedo-Bolton G, Lawton M, et al: Aneurysm growth occurs at region of low wall shear stress: patient-specific correlation of hemodynamics and growth in a longitudinal study. Stroke 39:29973002, 2008

6. Cebral JR, Castro MA, Burgess JE, Pergolizzi RS, Sheridan MJ, Putman CM: Characterization of cerebral aneurysms for assessing risk of rupture by using patient-specific computational hemodynamics models. AJNR Am J Neuroradiol 26:2550-2559, 2005

7. Cebral JR, Mut F, Weir J, Putman C: Quantitative characterization of the hemodynamic environment in ruptured and 
unruptured brain aneurysms. AJNR Am J Neuroradiol 32:145-151, 2011

8. Dhar S, Tremmel M, Mocco J, Kim M, Yamamoto J, Siddiqui $\mathrm{AH}$, et al: Morphology parameters for intracranial aneurysm rupture risk assessment. Neurosurgery 63:185-197, 2008

9. Fillinger MF, Marra SP, Raghavan ML, Kennedy FE: Prediction of rupture risk in abdominal aortic aneurysm during observation: wall stress versus diameter. J Vasc Surg 37:724-732, 2003

10. Ford MD, Alperin N, Lee SH, Holdsworth DW, Steinman DA: Characterization of volumetric flow rate waveforms in the normal internal carotid and vertebral arteries. Physiol Meas 26:477-488, 2005

11. Ford MD, Hoi Y, Piccinelli M, Antiga L, Steinman DA: An objective approach to digital removal of saccular aneurysms: technique and applications. Br J Radiol 82:S55-S61, 2009

12. Jou LD, Lee DH, Morsi H, Mawad ME: Wall shear stress on ruptured and unruptured intracranial aneurysms at the internal carotid artery. AJNR Am J Neuroradiol 29:1761-1767, 2008

13. Laaksamo E, Ramachandran M, Frösen J, Tulamo R, Baumann M, Friedlander RM, et al: Intracellular signaling pathways and size, shape, and rupture history of human intracranial aneurysms. Neurosurgery 70:1565-1573, 2012

14. Lauric A, Hippelheuser J, Cohen AD, Kadasi LM, Malek AM: Wall shear stress association with rupture status in volume matched sidewall aneurysms. J Neurointerv Surg 6:466-473, 2014

15. Lauric A, Miller EL, Baharoglu MI, Malek AM: 3D shape analysis of intracranial aneurysms using the writhe number as a discriminant for rupture. Ann Biomed Eng 39:14571469,2011

16. Lauric A, Miller EL, Baharoglu MI, Malek AM: Rupture status discrimination in intracranial aneurysms using the centroid-radii model. IEEE Trans Biomed Eng 58:28952903, 2011

17. Ma B, Harbaugh RE, Raghavan ML: Three-dimensional geometrical characterization of cerebral aneurysms. Ann Biomed Eng 32:264-273, 2004

18. Ma B, Lu J, Harbaugh RE, Raghavan ML: Nonlinear anisotropic stress analysis of anatomically realistic cerebral aneurysms. J Biomech Eng 129:88-96, 2007

19. Morita A, Kirino T, Hashi K, Aoki N, Fukuhara S, Hashimoto N, et al: The natural course of unruptured cerebral aneurysms in a Japanese cohort. N Engl J Med 366:2474-2482, 2012

20. Piccinelli M, Steinman DA, Hoi Y, Tong F, Veneziani A, Antiga L: Automatic neck plane detection and 3D geometric characterization of aneurysmal sacs. Ann Biomed Eng 40:2188-2211, 2012

21. Piccinelli M, Veneziani A, Steinman DA, Remuzzi A, Antiga L: A framework for geometric analysis of vascular structures: application to cerebral aneurysms. IEEE Trans Med Imaging 28: 1141-1155, 2009

22. Qian Y, Takao H, Umezu M, Murayama Y: Risk analysis of unruptured aneurysms using computational fluid dynamics technology: preliminary results. AJNR Am J Neuroradiol 32:1948-1955, 2011

23. Raghavan ML, Ma B, Harbaugh RE: Quantified aneurysm shape and rupture risk. J Neurosurg 102:355-362, 2005

24. Ramachandran M: On the Role of Intracranial Aneurysm Morphology in Stable Versus Unstable Lesions [dissertaton]. Iowa City, IA: University of Iowa, 2012

25. Ramachandran M, Laakso A, Harbaugh RE, Raghavan ML: On the role of modeling choices in estimation of cerebral aneurysm wall tension. J Biomech 45:2914-2919, 2012

26. Ramachandran M, Retarekar R, Harbaugh RE, Hasan D, Policeni B, Rosenwasser R, et al: Sensitivity of quantified intracranial aneurysm geometry to imaging modality. Cardiovasc Eng Technol 4:75-86, 2013
27. Retarekar R: Hemodynamics and Natural History Outcome in Unruptured Intracranial Aneurysms [dissertation]. Iowa City, IA: University of Iowa, 2012

28. Retarekar R, Ramachandran M, Berkowitz B, Harbaugh RE, Hasan D, Rosenwasser RH, et al: Stratification of a population of intracranial aneurysms using blood flow metrics. Comput Methods Biomech Biomed Engin 18:1072-1082, 2015

29. Saloner D, Martin A, Hurwit D, Sohrabi S, Lee A, Rayz V, et al: MRI/A in the evaluation of changes over time in untreated aneurysms. Proc Intl Soc Mag Reson Med 21:2995, 2013 (Abstract)

30. Shapiro SS, Wilk MB: An analysis of variance test for normality (complete samples). Biometrika 52:591-611, 1965

31. Shojima M, Oshima M, Takagi K, Torii R, Hayakawa M, Katada K, et al: Magnitude and role of wall shear stress on cerebral aneurysm: computational fluid dynamic study of 20 middle cerebral artery aneurysms. Stroke 35:2500-2505, 2004

32. Takao H, Murayama Y, Otsuka S, Qian Y, Mohamed A, Masuda $\mathrm{S}$, et al: Hemodynamic differences between unruptured and ruptured intracranial aneurysms during observation. Stroke 43:1436-1439, 2012

33. Tremmel M, Dhar S, Levy EI, Mocco J, Meng H: Influence of intracranial aneurysm-to-parent vessel size ratio on hemodynamics and implication for rupture: results from a virtual experimental study. Neurosurgery 64:622-631, 2009

34. Valen-Sendstad K, Piccinelli M, Steinman DA: High-resolution computational fluid dynamics detects flow instabilities in the carotid siphon: implications for aneurysm initiation and rupture? J Biomech 47:3210-3216, 2014

35. Valencia C, Villa-Uriol MC, Pozo JM, Frangi AF: Morphological descriptors as rupture indicators in middle cerebral artery aneurysms. Conf Proc IEEE Eng Med Biol Soc 2012:6046-6049, 2010

36. Wiebers DO, Whisnant JP, Huston J III, Meissner I, Brown RD Jr, Piepgras DG, et al: Unruptured intracranial aneurysms: natural history, clinical outcome, and risks of surgical and endovascular treatment. Lancet 362:103-110, 2003

37. Xiang J, Natarajan SK, Tremmel M, Ma D, Mocco J, Hopkins LN, et al: Hemodynamic-morphologic discriminants for intracranial aneurysm rupture. Stroke 42:144-152, 2011

\section{Disclosure}

The authors report no conflict of interest concerning the materials or methods used in this study or the findings specified in this paper.

\section{Author Contributions}

Conception and design: Harbaugh, Ramachandran, Retarekar, Raghavan, Ogilvy, Rosenwasser. Acquisition of data: Harbaugh, Ramachandran, Retarekar, Berkowitz, Dickerhoff, Correa, Lin, Johnson, Stapleton. Analysis and interpretation of data: Harbaugh, Ramachandran, Retarekar, Berkowitz, Dickerhoff, Correa, Lin, Torner. Drafting the article: Harbaugh, Ramachandran, Retarekar. Critically revising the article: Harbaugh, Ramachandran, Retarekar, Raghavan, Berkowitz, Dickerhoff, Hasan, Ogilvy, Rosenwasser, Bogason. Reviewed submitted version of manuscript: all authors. Approved the final version of the manuscript on behalf of all authors: Harbaugh. Statistical analysis: Harbaugh, Torner. Administrative/technical/material support: Stapleton. Study supervision: Harbaugh.

\section{Correspondence}

Robert E. Harbaugh, Department of Neurosurgery, Penn State Milton S. Hershey Medical Center, 30 Hope Dr., Hershey, PA 17033. email: reh1@mac.com. 